\title{
Aspectos de imagem na tendinite calcária pré-vertebral*
}

\author{
Imaging findings in acute calcific prevertebral tendinitis
}

\author{
Caio Giometti Grassi ${ }^{1}$, Fábio de Vilhena Diniz ${ }^{1}$, Márcio Ricardo Taveira Garcia², Regina Lúcia \\ Elia Gomes ${ }^{3}$, Mauro Miguel Daniel ${ }^{3}$, Marcelo Buarque de Gusmão Funari ${ }^{4}$
}

\begin{abstract}
Resumo A tendinite calcária aguda pré-vertebral é uma condição benigna e rara que apresenta calcificação das fibras do músculo longo do pescoço com reação inflamatória local, sendo esta uma das formas de apresentação menos frequentes da doença por deposição de hidroxiapatita de cálcio. Manifesta-se com dor cervical aguda e/ou odinofagia, podendo ser erroneamente diagnosticada como abscesso retrofaríngeo, espondilodiscite ou alteração decorrente de trauma. Os achados radiológicos na tendinite calcária pré-vertebral são patognomônicos. O conhecimento de tais achados é muito importante, pois o correto diagnóstico possibilita a resolução precoce dos sintomas e evita intervenções desnecessárias em um paciente que apresenta afecção com boa resposta ao tratamento conservador.

Unitermos: Tendinite calcária pré-vertebral; Músculo longo do pescoço; Doença por deposição de hidroxiapatita de cálcio; Imagem por ressonância magnética; Tomografia computadorizada.
\end{abstract}

\begin{abstract}
Acute calcific prevertebral tendinitis is a benign and rare condition that presents calcification of the superior oblique fibers of longus colli muscle with local inflammatory reaction. Such condition is one of the less common presentations of calcium hydroxyapatite deposition disease. Clinical signs are usually acute neck pain and odynophagia, and it may be misdiagnosed as retropharyngeal abscess, spondylodiscitis or traumatic injury. The imaging findings in calcific prevertebral tendinitis are pathognomonic. The knowledge of such findings is extremely important to avoid unnecessary interventions in a patient presenting a condition with a good response to conservative treatment.

Keywords: Calcific prevertebral tendinitis; Longus colli muscle; Calcium hydroxyapatite deposition disease; Magnetic resonance imaging; Computed tomography.

Grassi CG, Diniz FV, Garcia MRT, Gomes RLE, Daniel MM, Funari MBG. Aspectos de imagem na tendinite calcária pré-vertebral. Radiol Bras. 2011 Set/Out;44(5):327-330.
\end{abstract}

\section{INTRODUÇÃO}

Tendinite calcária aguda pré-vertebral, ou tendinite calcária aguda retrofaríngea, é uma síndrome clínica que foi originalmente descrita por Hartley em 1964. Em 1994, Ring demonstrou que a síndrome é decorrente da deposição de hidroxiapatita de cálcio no músculo longo do pescoço, um flexor cervical que se localiza no espaço pré-vertebral $^{(1-4)}$.

* Trabalho realizado no Departamento de Imagem do Hospital Israelita Albert Einstein, São Paulo, SP, Brasil.

1. Médicos Residentes do Departamento de Imagem do Hospital Israelita Albert Einstein, São Paulo, SP, Brasil.

2. Médico Radiologista do Departamento de Imagem do Hospital Israelita Albert Einstein, São Paulo, SP, Brasil.

3. Doutores, Médicos Radiologistas do Departamento de Imagem do Hospital Israelita Albert Einstein, São Paulo, SP, Brasil.

4. Doutor, Médico Radiologista, Coordenador do Departamento de Imagem do Hospital Israelita Albert Einstein, São Paulo, SP, Brasil.

Endereço para correspondência: Dr. Caio Giometti Grassi. Hospital Israelita Albert Einstein - Departamento de Imagem, Sala de Laudos. Avenida Albert Einstein, 627, $4^{\circ}$ andar, Bloco D, Morumbi. São Paulo, SP, Brasil, 05652-901. E-mail: caiograss @hotmail.com

Recebido para publicação em 10/9/2010. Aceito, após revisão, em 20/6/2011.
Apresentamos dois casos de tendinite pré-vertebral, com ênfase nos achados de imagem.

\section{Caso 1}

Paciente do sexo masculino, 39 anos de idade, com queixa de cefaleia occipital, dor e limitação da movimentação cervical há três dias, sem queda do estado geral ou febre. Antecedentes patológicos e cirúrgicos negativos. Ao exame físico foram observados sinais gerais normais, redução da amplitude de movimentação cervical, rigidez de nuca e sinal de Brudzinski positivo. A hipótese diagnóstica inicial foi de meningismo a esclarecer, tendo sido solicitados exames laboratoriais gerais e líquor, os quais estavam normais, com exceção da proteína C-reativa, que era de 13 (valor de referência: 0-3,0). Foram solicitadas radiografia cervical (Figura 1), que mostrava calcificação na região pré-vertebral ao nível de C1-C2, e em seguida tomografia computadorizada cervical (Figura 1), evidenciandose calcificação no músculo longo do pes- coço com aumento de partes moles adjacentes e líquido no espaço retrofaríngeo, sugerindo tendinite calcária aguda pré-vertebral. Foi solicitada ressonância magnética de pescoço (Figura 2) para melhor avaliação do líquido no espaço retrofaríngeo, não tendo havido realce local após a administração do contraste, ratificando o diagnóstico.

\section{Caso 2}

Paciente do sexo masculino, 47 anos de idade, com dor à movimentação cervical e odinofagia há quatro dias, sem queda do estado geral ou febre. Antecedentes patológicos e cirúrgicos negativos. Ao exame físico constatou-se redução da amplitude de movimentação cervical. Demais aspectos sem particularidades. Exames laboratoriais gerais sem alterações. Foi solicitada tomografia computadorizada de pescoço (Figura 3), com achados sugestivos de tendinite aguda pré-vertebral. Exame de ressonância magnética cervical (Figura 4) posteriormente corroborou o diagnóstico. 

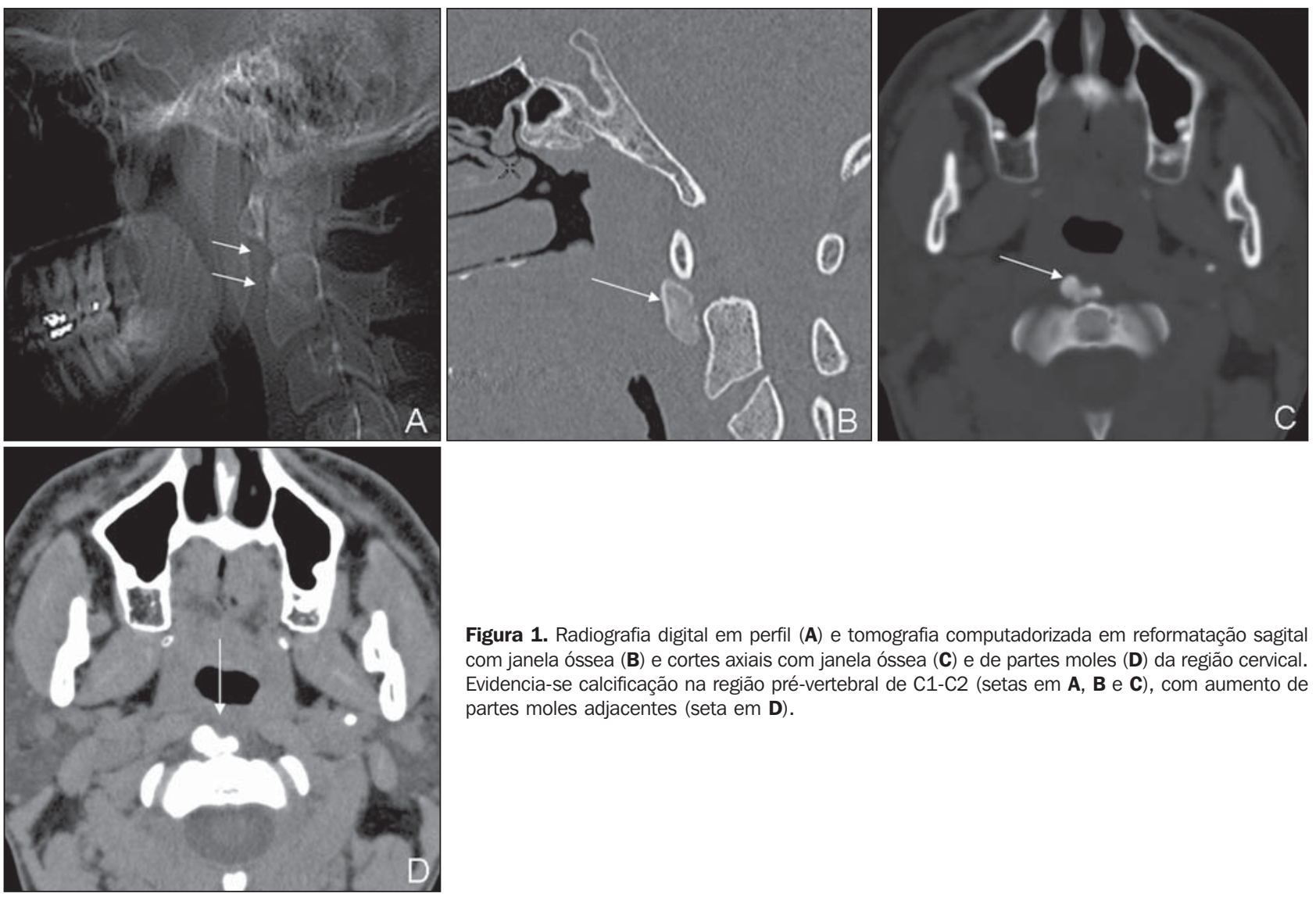

Figura 1. Radiografia digital em perfil (A) e tomografia computadorizada em reformatação sagital com janela óssea (B) e cortes axiais com janela óssea (C) e de partes moles (D) da região cenvical. Evidencia-se calcificação na região pré-vertebral de C1-C2 (setas em A, B e C), com aumento de partes moles adjacentes (seta em $\mathbf{D}$ ).
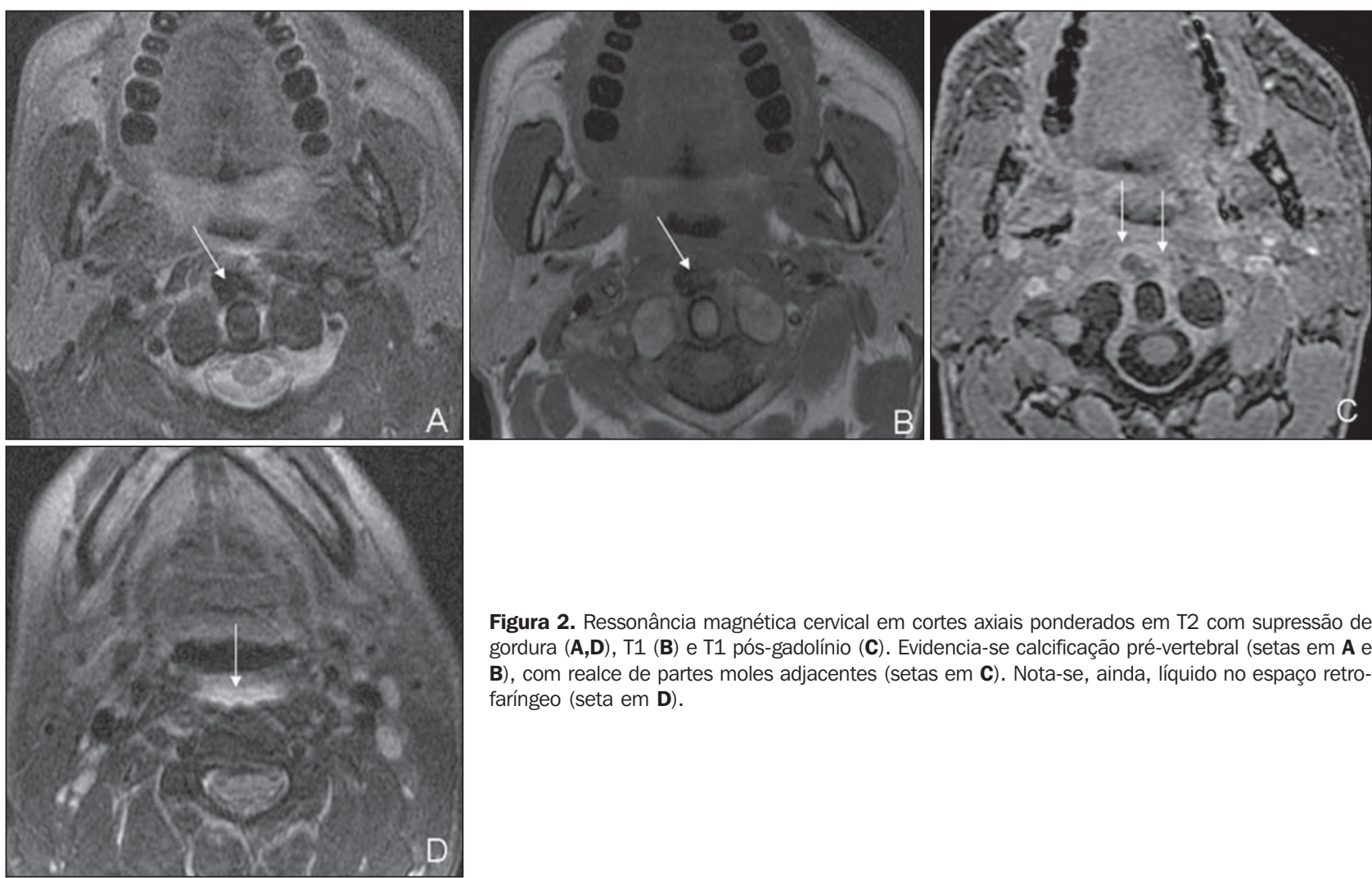

Figura 2. Ressonância magnética cervical em cortes axiais ponderados em T2 com supressão de gordura (A,D), T1 (B) e T1 pós-gadolínio (C). Evidencia-se calcificação pré-vertebral (setas em $\mathbf{A}$ B), com realce de partes moles adjacentes (setas em C). Nota-se, ainda, líquido no espaço retrofaríngeo (seta em $\mathbf{D}$ ). 

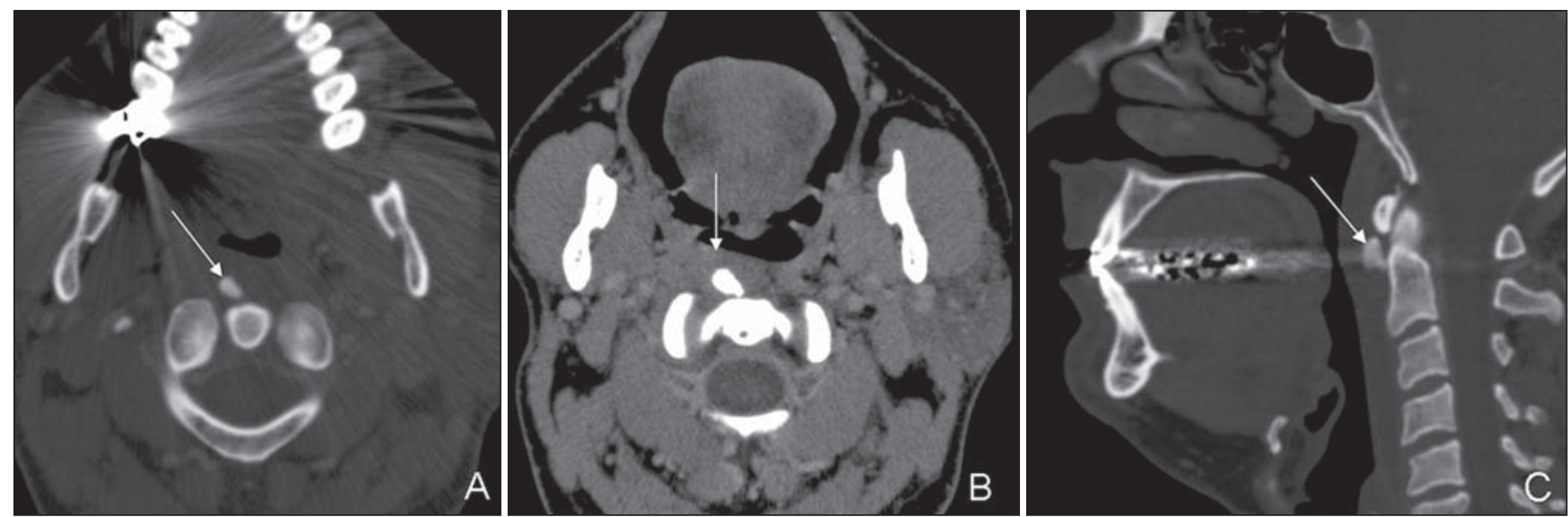

Figura 3. Tomografia computadorizada de pescoço em cortes axiais $(\mathbf{A}, \mathbf{B})$ e reformatações sagitais (C,D), com janela óssea (A,C) e de partes moles (B,D). Observa-se calcificação pré-vertebral ao nível de C1-C2 no músculo longo do pescoço (seta em $\mathbf{A}$ e $\mathbf{C}$ ), bem como aumento de partes moles adjacentes (seta em B) e líquido no espaço retrofaríngeo (seta em D).
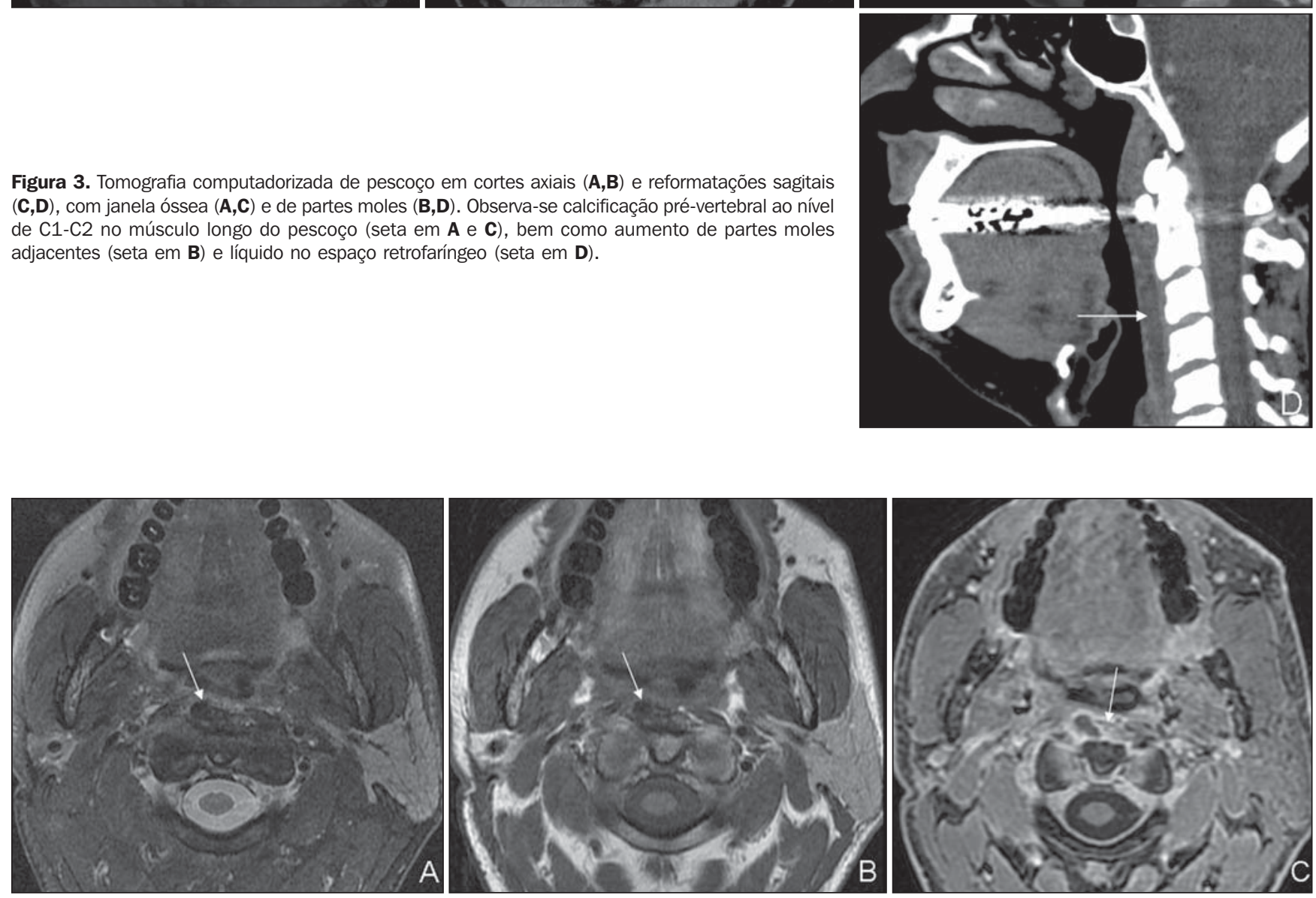

Figura 4. Ressonância magnética de pescoço com imagens axiais ponderadas em $T 2$ com saturação de gordura (A), T1 (B) e T1 pós-gadolínio (C), e imagem sagital ponderada em T2 com saturação de gordura (D). Notam-se calcificação no músculo longo do pescoço à direita (setas em $\mathbf{A}$ e $\mathbf{B}$ ), realce de partes moles adjacentes (seta em C) e líquido no espaço retrofaríngeo (seta em D).

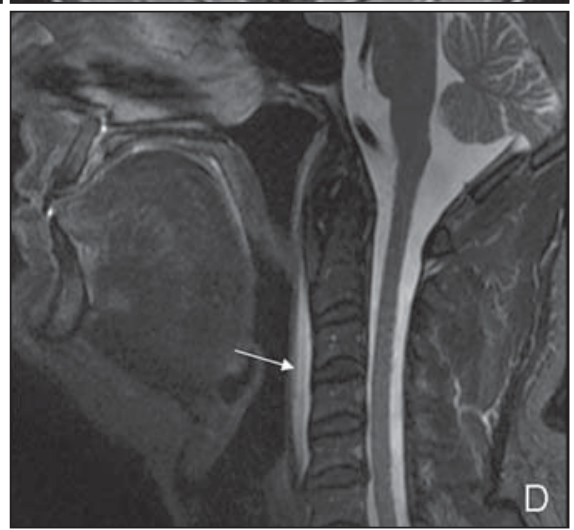




\section{DISCUSSÃO}

A tendinite do músculo longo do pescoço ocorre predominantemente em indivíduos na faixa etária entre 30 e 60 anos e é uma das formas de apresentação menos frequentes da doença por depósito de cristais de hidroxiapatita de cálcio ${ }^{(5,6)}$.

As manifestações clínicas são geralmente de dor cervical aguda e/ou de odinofagia. Ao exame físico pode haver dor à palpação e redução da amplitude de movimentação cervical ${ }^{(\mathbf{3 , 4}, 7)}$.

Em relação ao diagnóstico por imagem, os achados da radiografia cervical em perfil são tidos como patognomônicos e consistem em calcificação pré-vertebral ao nível de C1-C2 com edema de partes moles adjacentes ${ }^{(2,7,8)}$

$\mathrm{O}$ aumento de partes moles pré-vertebrais pode se estender de $\mathrm{C} 1$ a $\mathrm{C} 4$ e ter espessura tão grande quanto 1,5 a $2,0 \mathrm{~cm}^{(\mathbf{( 9 )}}$.

A maior resolução de contraste da tomografia computadorizada faz com que esta apresente maior sensibilidade em relação à radiografia simples na detecção da calcificação pré-vertebral e do edema de partes moles. Além disso, permite a identificação de edema retrofaríngeo, se presente ${ }^{(8)}$.

A ressonância magnética tem menor sensibilidade na identificação da calcificação pré-vertebral, que apresenta baixo sinal nas imagens ponderadas em $\mathrm{T} 1$ e $\mathrm{T} 2{ }^{(6)}$.
Pode-se lançar mão da sequência gradiente eco $(\mathrm{T} 2 *)$ para aumentar a sensibilidade na detecção da calcificação, que se apresenta com marcado hipossinal.

Graças à sua maior resolução de contraste, a ressonância magnética tem maior acurácia na caracterização do edema de partes moles e retrofaríngeo ${ }^{(\mathbf{6})}$.

Na tendinite calcária pré-vertebral pode ser detectado líquido no espaço retrofaríngeo em exames de imagem. Tal achado pode ser confundido com abscesso retrofaríngeo de origem infecciosa. Para diferenciar tais condições, é importante observar que na infecção retrofaríngea encontrase um quadro infeccioso grave, bem como realce periférico do espaço retrofaríngeo nas imagens pós-contraste, enquanto na tendinite calcária pré-vertebral não há sinais clínicos de infecção, tampouco realce retrofaríngeo $^{(4)}$.

Se não tratada, a tendinite calcária se resolve espontaneamente em algumas semanas. No entanto, o diagnóstico e o tratamento sintomático precoces são muito importantes, pois o quadro álgico é debilitante neste período sem tratamento ${ }^{(\mathbf{5})}$.

O tratamento consiste em anti-inflamatórios por duas semanas, usualmente com resolução dos sintomas em 72 horas $^{(\mathbf{5})}$.

Concluímos que o conhecimento dos achados de imagem na tendinite pré-vertebral é muito importante, pois o correto diag- nóstico possibilita tratamento sintomático em tempo hábil e evita intervenções desnecessárias em um paciente que apresenta afecção com boa resposta à abordagem conservadora.

\section{REFERÊNCIAS}

1. Hartley J. Acute cervical pain associated with retropharyngeal calcium deposit: a case report. J Bone Joint Surg Am. 1964;46:1753-4.

2. Ring D, Vaccaro AR, Scuderi G, et al. Acute calcific retropharyngeal tendinitis. Clinical presentation and pathological characterization. J Bone Joint Surg Am. 1994;76:1636-42.

3. Razon RVB, Nasir A, Wu GS, et al. Retropharyngeal calcific tendonitis: report of two cases. J Am Board Fam Med. 2009;22:84-8.

4. Eastwood JD, Hudgins PA, Malone D. Retropharyngeal effusion in acute calcific prevertebral tendinitis: diagnosis with CT and MR imaging. AJNR Am J Neuroradiol. 1998;19:1789-92.

5. Hayes CW, Conway WF. Calcium hydroxyapatite deposition disease. Radiographics. 1990;10:103148.

6. Mihmanli I, Karaarslan E, Kanberoglu K. Inflammation of vertebral bone associated with acute calcific tendinitis of the longus colli muscle. Neuroradiology. 2001;43:1098-101.

7. Kaplan MJ, Eavey RD. Calcific tendinitis of the longus colli muscle. Ann Otol Rhinol Laryngol. 1984;93(3 Pt 1):215-9.

8. Artenian DJ, Lipman JK, Scidmore GKI, et al Acute neck pain due to tendonitis of the longus colli: CT and MRI findings. Neuroradiology. 1989;31:166-9.

9. Haun CL. Retropharyngeal tendinitis. AJR Am J Roentgenol. 1978;130:1137-40. 